International Journal of Pure and Applied Mathematics

Volume 94 No. 2 2014, 277-293

ISSN: 1311-8080 (printed version); ISSN: 1314-3395 (on-line version)

url: http://www.ijpam.eu

doi: http://dx.doi.org/10.12732/ijpam.v94i2.14

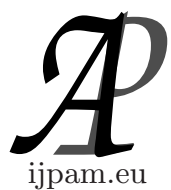

\title{
POSITIVE SOLUTIONS OF TWO-POINT BOUNDARY VALUE PROBLEMS FOR SECOND ORDER IMPULSIVE DIFFERENTIAL EQUATIONS
}

\author{
Ying $\mathrm{He}$ \\ School of Mathematics and Statistics \\ Northeast Petroleum University \\ Daqing, 163318, P.R. CHINA
}

\begin{abstract}
In this paper we consider the existence of positive solutions of twopoint boundary value problems for second-order nonlinear impulsive differential equations. By constructing a cone $K_{1} \times K_{2}$, which is the Cartesian product of two cones in the space $\mathrm{C}[0,1]$ and computing the fixed point index in the $K_{1} \times K_{2}$, we establish the existence of positive solutions for the system.
\end{abstract}

AMS Subject Classification: 34B15

Key Words: singular two-point boundary value problem with impulsive terms, second-order impulsive differential equations, fixed point index in cones

\section{Introduction}

The theory of impulsive differential equations has been emerging as an important area of investigation in recent years. For some general and recent works on the theory of impulsive differential equations we refer the reader to [2-4]. Applications of impulsive differential equations occur in biology, medicine, engineering. In this paper,we consider the existence of positive solutions for the

Received: May 22, 2014

(c) 2014 Academic Publications, Ltd. url: www.acadpubl.eu 
following second-order impulsive differential equations:

$$
\left\{\begin{array}{l}
-L u=h_{1}(x) g_{1}(x, u, v), \quad x \in I^{\prime}, \\
-L v=h_{2}(x) g_{2}(x, v, u), \quad x \in I^{\prime}, \\
-\left.\Delta\left(p u^{\prime}\right)\right|_{x=x_{k}}=I_{1, k}\left(u\left(x_{k}\right)\right), \quad k=1,2, \cdots, m, \\
-\left.\Delta\left(p v^{\prime}\right)\right|_{x=x_{k}}=I_{2, k}\left(v\left(x_{k}\right)\right), \quad k=1,2, \cdots, m, \\
\alpha u(0)-\beta u^{\prime}(0)=0, \quad \alpha v(0)-\beta v^{\prime}(0)=0, \\
\gamma u(1)+\delta u^{\prime}(1)=0, \quad \gamma v(1)+\delta v^{\prime}(1)=0
\end{array}\right.
$$

here $L u=\left(p(x) u^{\prime}\right)^{\prime}+q(x) u$ is sturm-liouville operator, $L v=\left(p(x) v^{\prime}\right)^{\prime}+q(x) v$ is also sturm-liouville operator, $I=[0,1], I^{\prime}=I \backslash\left\{x_{1}, x_{2}, \cdots, x_{m}\right\}$ and $0<$ $x_{1}<x_{2}<\cdots<x_{m}<1$ are given, $R^{+}=[0,+\infty), g \in C\left(I \times R^{+}, R^{+}\right), I_{k}, \bar{I}_{k} \in$ $C\left(R^{+}, R^{+}\right),\left.\Delta\left(p u^{\prime}\right)\right|_{x=x_{k}}=p\left(x_{k}\right) u^{\prime}\left(x_{k}^{+}\right)-p\left(x_{k}\right) u^{\prime}\left(x_{k}^{-}\right),\left.\quad \Delta\left(p v^{\prime}\right)\right|_{x_{1} x_{k}}=$ $p\left(x_{k}\right) v^{\prime}\left(x_{k}^{+}\right)-p\left(x_{k}\right) v^{\prime}\left(x_{k}^{-}\right), u^{\prime}\left(x_{k}^{+}\right), v^{\prime}\left(x_{k}^{+}\right)\left(u^{\prime}\left(x_{k}^{-}\right), v^{\prime}\left(x_{k}^{-}\right)\right)$denote the right limit ( left limit) of $u^{\prime}(x)$ and $v^{\prime}(x)$ at $x=x_{k}$ respectively, $h_{i}(x) \in C(I,(0, \propto))(i=$ 1,2 ) and may be singular at $x=0$ or $x=1$.

Throughout this paper, we always suppose that

$\left(S_{1}\right) p(x) \in C^{1}([0,1], R), p(x)>0, q(x) \in C([0,1], R), q(x) \leq 0, \alpha, \beta, \gamma, \delta \geq$ $0, \rho=\beta \gamma+\alpha \gamma+\alpha \delta>0$.

In recent yeas,many authors have studied existence of positive radial solutions for elliptic systems, which is equivalent to that of positive solutions for corresponding ordinary differential systems,see [5-9] and the references therein. The usual method used is applying a fixed point theorem of a cone expansion and compression or the fixed point index theory in cones. However most of ordinary differential equations with no singularity and impulse. Motivated by the work mentioned above,in this paper,we study the existence of positive solutions of two-point boundary value problems for the nonlinear second-order singular and impulsive differential equation system(1.1). By constructing a cone $K \times K$, which is the Cartesian product of two cones in the space $\mathrm{C}[0,1]$, and computing the fixed-point index in the $K \times K$ under some conditions on $h_{i}(x)$ concerning the first eigenvalue corresponding to the relevant linear operator, we establish the existence of positive solutions for the singular and impulsive differential system (1.1).

\section{Preliminary}

Let $Q=I \times I$ and $Q_{1}=\{(x, y) \in Q \mid 0 \leq x \leq y \leq 1\}, Q_{2}=\{(x, y) \in Q \mid 0 \leq$ $y \leq x \leq 1\}$. Let $G(x, y)$ is the Green's function of the boundary value problem

$$
-L u=0, R_{1}(u)=R_{2}(u)=0 .
$$


Following from [4], $G(x, y)$ can be written by

$$
G(x, y):= \begin{cases}\frac{m(x) n(y)}{\omega}, & (x, y) \in Q_{1}, \\ \frac{m(y) n(x)}{\omega}, & (x, y) \in Q_{2} .\end{cases}
$$

Lemma 2.1. (see [4]) Suppose that $\left(S_{1}\right)$ holds, then the Green's function $G(x, y)$, defined by (2.1), possesses the following properties:

(i): $m(x) \in C^{2}(I, R)$ is increasing and $m(x)>0, x \in(0,1]$.

(ii): $n(x) \in C^{2}(I, R)$ is decreasing and $n(x)>0, x \in[0,1)$.

(iii): $(\operatorname{Lm})(x) \equiv 0, m(0)=\beta, m^{\prime}(0)=\alpha$.

(iv): $(\operatorname{Ln})(x) \equiv 0, n(1)=\delta, n^{\prime}(1)=-\gamma$.

(v): $\omega$ is a positive constant.Moreover, $p(x)\left(m^{\prime}(x) n(x)-m(x) n^{\prime}(x)\right) \equiv \omega$.

(vi): $G(x, y)$ is continuous and symmetrical over $Q$.

(vii): $G(x, y)$ has continuously partial derivative over $Q_{1}, Q_{2}$.

(viii): For each fixed $y \in I, G(x, y)$ satisfies $L G(x, y)=0$ for $x \neq y, x \in I$. Moreover, $R_{1}(G)=R_{2}(G)=0$ for $y \in(0,1)$.

(viii): $G_{x}^{\prime}$ has discontinuous point of the first kind at $x=y$ and

$$
G_{x}^{\prime}(y+0, y)-G_{x}^{\prime}(y-0, y)=-\frac{1}{p(y)}, y \in(0,1) .
$$

Following from Lemma2.1, it is easy to see that:

$$
\begin{gathered}
G(x, y) \leq G(y, y)=\frac{m(y) n(y)}{\omega}, \quad x, y \in[0,1], \\
G(x, y) \geq \sigma G(y, y), x \in[a, b], y \in[0,1], \text { where } a \in\left(0, t_{1}\right], b \in\left[t_{m}, 1\right), \\
0<\sigma=\min \left\{\frac{m(a)}{m(1)}, \frac{n(b)}{n(0)}\right\}<1
\end{gathered}
$$

We denote the first eigenvalue of

$$
-L \phi=\lambda \phi h_{1}, \alpha \phi(0)-\beta \phi^{\prime}(0)=0, \gamma \phi(1)+\delta \phi^{\prime}(1)=0
$$

by $\lambda_{1}$ and the corresponding eigenfunction by $\phi_{1}(x)$. It is well-known that $\lambda_{1}>0$, and $\phi_{1}(x)>0$ for $x \in(0,1)$. 
In the same way, for

$$
-L \phi=\lambda \phi h_{2}, \alpha \phi(0)-\beta \phi^{\prime}(0)=0, \gamma \phi(1)+\delta \phi^{\prime}(1)=0,
$$

we get $\mu_{1}>0$ as the first eigenvalue and the corresponding eigenfunction by $\phi_{2}(x)$, satisfying $\phi_{2}(x)>0$ for $x \in(0,1)$.

To conclude the introduction,we introduce the following notation:

$$
\begin{gathered}
g_{i, 0}(v)=\liminf _{u \rightarrow 0^{+}} \min _{x \in[a, b]} \frac{g_{i}(x, u, v)}{u}, I_{i, 0}(k)=\liminf _{u \rightarrow 0^{+}} \frac{I_{i, k}(u)}{u} \\
g_{i, \infty}(v)=\liminf _{u \rightarrow+\infty} \min _{x \in[a, b]} \frac{g_{i}(x, u, v)}{u}, I_{i, \infty}(k)=\liminf _{u \rightarrow+\infty} \frac{I_{i, k}(u)}{u}, \\
g_{i}^{\infty}(v)=\limsup _{u \rightarrow+\infty} \max _{x \in[a, b]} \frac{g_{i}(x, u, v)}{u}, I_{i}^{\infty}(k)=\limsup _{u \rightarrow+\infty} \frac{I_{i, k}(u)}{u}, \\
g_{i}^{0}(v)=\limsup _{u \rightarrow 0^{+}} \max _{x \in[a, b]} \frac{g_{i}(x, u, v)}{u}, I_{i}^{0}(k)=\limsup _{u \rightarrow 0^{+}} \frac{I_{i, k}(u)}{u} .
\end{gathered}
$$

where $v \in R^{+}$and $i=1,2$.

Moreover, for the simplicity in the following discussion, we introduce the following hypotheses.

$\left(H_{1}\right)$ :

$\inf _{z \in R^{+}} g_{1,0}(z)+\frac{\sigma \sum_{k=1}^{m} I_{1,0}(k) \phi_{1}\left(x_{k}\right)}{\int_{a}^{b} \phi_{1}(x) h_{1}(x) d x}>\lambda_{1}, \sup _{z \in R^{+}} g_{1}^{\infty}(z)+\frac{\sum_{k=1}^{m} I_{1}^{\infty}(k) \phi_{1}\left(x_{k}\right)}{\int_{a}^{b} \sigma \phi_{1}(x) h_{1}(x) d x}<\lambda_{1}$.

$\left(H_{2}\right)$ :

$\sup _{z \in R^{+}} g_{2}^{0}(z)+\frac{\sum_{k=1}^{m} I_{2}^{0}(k) \phi_{2}\left(x_{k}\right)}{\int_{a}^{b} \sigma \phi_{2}(x) h_{2}(x) d x}<\mu_{1}, \inf _{z \in R^{+}} g_{2, \infty}(z)+\frac{\sigma \sum_{k=1}^{m} I_{2, \infty}(k) \phi_{2}\left(x_{k}\right)}{\int_{a}^{b} \phi_{2}(x) h_{2}(x) d x}>\mu_{1}$.

$\left(H_{3}\right): 0<\int_{0}^{1} G(y, y) h_{1}(y) d y<+\infty \quad 0<\int_{0}^{1} G(y, y) h_{2}(y) d y<+\infty$

In this paper, we shall consider the following space

$$
\begin{array}{r}
P C^{\prime}(I, R)=\left\{u \in C(I, R) ;\left.u^{\prime}\right|_{\left(x_{k}, x_{k+1}\right)} \in C\left(x_{k}, x_{k+1}\right), u^{\prime}\left(x_{k}^{-}\right)=u^{\prime}\left(x_{k}\right),\right. \\
\left.\exists u^{\prime}\left(x_{k}^{+}\right), \quad k=1,2, \cdots, m\right\},
\end{array}
$$


with the norm $\|u\|_{P C^{\prime}}=\max \left\{\|u\|,\left\|u^{\prime}\right\|\right\}$, here $\|u\|=\sup _{x \in[0,1]}|u(x)|,\left\|u^{\prime}\right\|=$ $\sup _{x \in[0,1]}\left|u^{\prime}(x)\right|$. Then $P C^{\prime}(I, R)$ is a Banach space.

Definition 2.1. A couple function

$$
(u, v) \in P C^{\prime}(I, R) \cap C^{2}\left(I^{\prime}, R\right) \times P C^{\prime}(I, R) \cap C^{2}\left(I^{\prime}, R\right)
$$

is called a solution of system (1.1) if it satisfies system (1.1)

In applications below, we take $E=C(I, R)$ and define

$$
K=\{u \in C(I, R): u(x) \geq \sigma\|u\|, x \in[a, b]\} .
$$

One may readily verify that $K$ is a closed convex cone in $E$. For $r>0$, let $K_{r}=\{u \in K:\|u\|<r\}$ and $\partial K_{r}=\{u \in K:\|u\|=r\}$. For any $(u, v) \in K \times K$,define mappings $\Phi_{v}: K \rightarrow C\left(I, R^{+}\right), \Psi_{u}: K \rightarrow C\left(I, R^{+}\right)$, and $T: K \times K \rightarrow C\left(I, R^{+}\right) \times C\left(I, R^{+}\right)$as follows

$$
\begin{aligned}
\Phi_{v}(u)(x)= & \int_{0}^{1} G(x, y) h_{1}(y) g_{1}(y, u(y), v(y)) d y \\
& +\sum_{0<x_{k}<x} G\left(x, x_{k}\right) I_{1, k}\left(u\left(x_{k}\right)\right), x \in I . \\
\Psi_{u}(v)(x)= & \int_{0}^{1} G(x, y) h_{2}(y) g_{2}(y, v(y), u(y)) d y \\
& +\sum_{0<x_{k}<x} G\left(x, x_{k}\right) I_{2, k}\left(v\left(x_{k}\right)\right), x \in I \\
T(u, v)(x)= & \left(\Phi_{v}(u)(x), \Psi_{u}(v)(x)\right), \quad x \in[0,1] .
\end{aligned}
$$

Form $\left(H_{3}\right)$,we know that $\Phi_{v}$ and $\Psi_{u}$ are well-defined.And so $\mathrm{T}$ is well defined. We need the following lemmas in this paper.

Lemma 2.2. The vector $(u, v) \in P C^{\prime}(I, R) \cap C^{2}\left(I^{\prime}, R\right) \times P C^{\prime}(I, R) \cap$ $C^{2}\left(I^{\prime}, R\right)$ is a solution of differential system (1.1)if and only if $(u, v) \in P C^{\prime}(I, R) \times$ $P C^{\prime}(I, R)$ is a solution of the following integral system

$$
\left\{\begin{array}{l}
u(x)=\int_{0}^{1} G(x, y) h_{1}(y) g_{1}(y, u(y), v(y)) d y+\sum_{0<x_{k}<x} G\left(x, x_{k}\right) I_{1, k}\left(u\left(x_{k}\right)\right), \\
v(x)=\int_{0}^{1} G(x, y) h_{2}(y) g_{2}(y, v(y), u(y)) d y+\sum_{0<x_{k}<x} G\left(x, x_{k}\right) I_{2, k}\left(v\left(x_{k}\right)\right) .
\end{array}\right.
$$


Lemma 2.3. If $\left(H_{3}\right)$ is satisfied, then $T: K \times K \rightarrow K \times K$ is completely continuous. Moreover, $T(K \times K) \subset K \times K$.

Proof. It is easy to see that $T: K \times K \rightarrow K \times K$ is completely continuous. Thus we only need to show $T(K \times K) \subset K \times K$

In fact, by using inequalities $(2.2)$ and $\left(H_{3}\right)$, we have that

$$
\begin{aligned}
\left\|\Phi_{v}(u)\right\| \leq \int_{0}^{1} G(y, y) h_{1}(y) g_{1}(y, u(y), v(y)) d y & \\
& +\sum_{0<x_{k}<x} G\left(x_{k}, x_{k}\right) I_{1, k}\left(u\left(x_{k}\right)\right)<+\infty, \\
\left\|\Psi_{u}(v)\right\| \leq \int_{0}^{1} G(y, y) h_{2}(y) g_{2}(y, v(y), u(y)) d y & \\
& +\sum_{0<x_{k}<x} G\left(x_{k}, x_{k}\right) I_{2, k}\left(v\left(x_{k}\right)\right)<+\infty .
\end{aligned}
$$

On the other hand,for any $x \in[a, b]$, we obtain

$$
\begin{aligned}
\Phi_{v}(u)(x) & =\int_{0}^{1} G(x, y) h_{1}(y) g_{1}(y, u(y), v(y)) d y+\sum_{0<x_{k}<x} G\left(x, x_{k}\right) I_{1, k}\left(u\left(x_{k}\right)\right) \\
\geq & \int_{a}^{b} G(x, y) h_{1}(y) g_{1}(y, u(y), v(y)) d y+\sum_{0<x_{k}<x} G\left(x, x_{k}\right) I_{1, k}\left(u\left(x_{k}\right)\right) \\
\geq & \sigma\left(\int_{0}^{1} G(y, y) h_{1}(y) g_{1}(y, u(y), v(y)) d y\right. \\
& +\sum_{0<x_{k}<x} G\left(x_{k}, x_{k}\right) I_{1, k}\left(u\left(x_{k}\right)\right) \\
\geq & \sigma\left\|\Phi_{v}(u)\right\| .
\end{aligned}
$$

Similarly, $\Psi_{u}(v)(x) \geq \sigma\left\|\Psi_{u}(v)\right\|$. Thus, $\Phi_{v}(u)(x) \in K$ and $\Psi_{u}(v)(x) \in K$. Consequently, $T(K \times K) \subset K \times K$.

Lemma 2.4. Let $\Phi: K \rightarrow K$ be a completely continuous mapping with $\mu \Phi u \neq u$ for every $u \in \partial K_{r}$ and $0<\mu \leq 1$. Then $i\left(\Phi, K_{r}, K\right)=1$.

Lemma 2.5. Let $\Phi: K \rightarrow K$ be a completely continuous mapping. Suppose that the following two conditions are satisfied: 
(i) $\inf _{u \in \partial K_{r}}\|\Phi u\|>0$;

(ii) $\mu \Phi u \neq u$ for every $u \in \partial K_{r}$ and $\mu \geq 1$.

Then, $i\left(\Phi, K_{r}, K\right)=0$.

Lemma 2.6. Let $E$ be a Banach space and $K_{i} \subset K(i=1,2)$ be a closed set in $E$. For $r_{i}>0(i=1,2)$, denote $K_{r_{i}}=\left\{u \in K_{i}:\|u\|<r_{i}\right\}$ and $\partial K_{r_{i}}=\left\{u \in K_{i}:\|u\|=r_{i}\right\}$. Suppose $\Phi_{i}: K_{i} \rightarrow K_{i}$ is completely continuous. If $u_{i} \neq \Phi_{i} u_{i}$ for any $u_{i} \in \partial K_{r_{i}}$, then

$$
i\left(\Phi, K_{r_{1}} \times K_{r_{2}}, K_{1} \times K_{2}\right)=i\left(\Phi_{1}, K_{r_{1}}, K_{1}\right) \times i\left(\Phi_{2}, K_{r_{2}}, K_{2}\right)
$$

where $\Phi(u, v)=:\left(\Phi_{1} u, \Phi_{2} v\right)$ for any $(u, v) \in K_{1} \times K_{2}$.

\section{Main Results}

Theorem 3.1. Assume that $\left(H_{1}\right)-\left(H_{3}\right)$ are satisfied. Then problem (1.1) has at least one positive solution $(u, v)$.

To prove Theorem 3.1, we first give the following lemmas.

Lemma 3.1. If $\left(H_{1}\right)$ and $\left(H_{3}\right)$ are satisfied, then $i\left(\Phi_{v}, K_{R_{1}} \backslash \bar{K}_{r_{1}}, K\right)=1$.

Proof. Since $\left(H_{1}\right)$ holds, then there exists $0<\varepsilon<\min \left\{1, \lambda_{1}-\sup _{z \in R^{+}} g_{1}^{\infty}(z)\right\}$ such that

$$
\begin{gathered}
(1-\varepsilon)\left[\inf _{z \in R^{+}} g_{1,0}(z)+\frac{\sigma \sum_{k=1}^{m} I_{1,0}(k) \phi_{1}\left(x_{k}\right)}{\int_{a}^{b} \phi_{1}(x) h_{1}(x) d x}\right]>\lambda_{1}, \\
\left(\lambda_{1}-\varepsilon-\sup _{z \in R^{+}} g_{1}^{\infty}(z)\right) \int_{a}^{b} \sigma \phi_{1}(x) h_{1}(x) d x>\sum_{k=1}^{m}\left(I_{1}^{\infty}(k)+\varepsilon\right) \phi_{1}\left(x_{k}\right) .
\end{gathered}
$$

By the definitions of $g_{1,0}, I_{1,0}$, one can find $r_{0}>0$ such that

$$
\begin{aligned}
& g_{1}(x, u, v) \geq g_{1,0}(v)(1-\varepsilon) u, I_{1, k}(u) \geq I_{1,0}(k)(1-\varepsilon) u, \\
& \forall x \in[a, b], 0<u<r_{0}, v \in R^{+} .
\end{aligned}
$$

Let $r_{1} \in\left(0, r_{0}\right)$, then for $u \in \partial K_{r_{1}}$, we have

$$
u(x) \geq \sigma\|u\|=\sigma r_{1}>0 . \quad \forall x \in[a, b]
$$


Thus

$$
\begin{aligned}
\Phi_{v} u\left(\frac{1}{2}\right)= & \int_{0}^{1} G\left(\frac{1}{2}, y\right) h_{1}(y) g_{1}(y, u(y), v(y)) d y+\sum_{0<x_{k}<\frac{1}{2}} G\left(\frac{1}{2}, x_{k}\right) I_{1, k}\left(u\left(x_{k}\right)\right) \\
\geq & \int_{a}^{b} G\left(\frac{1}{2}, y\right) h_{1}(y) g_{1}(y, u(y), v(y)) d y+\sum_{0<x_{k}<\frac{1}{2}} G\left(\frac{1}{2}, x_{k}\right) I_{1, k}\left(u\left(x_{k}\right)\right) \\
\geq & (1-\varepsilon) \int_{a}^{b} G\left(\frac{1}{2}, y\right) h_{1}(y) g_{1,0}(v(y)) u(y) d y \\
& +(1-\varepsilon) \sum_{0<x_{k}<\frac{1}{2}} G\left(\frac{1}{2}, x_{k}\right) I_{1,0}(k) u\left(x_{k}\right) \\
\geq & (1-\varepsilon) \sigma r_{1}\left(\inf _{z \in R^{+}} g_{1,0}(z) \int_{a}^{b} G\left(\frac{1}{2}, y\right) h_{1}(x) d y\right. \\
& +\left.\sum_{0<x_{k}<\frac{1}{2}} G\left(\frac{1}{2}, x_{k}\right) I_{1,0}(k)\right|_{>}
\end{aligned}
$$

from which we see that $\inf _{u \in \partial K_{r_{1}}}\left\|\Phi_{v} u\right\|>0$, namely, hypothesis (i) of Lemma 2.5 holds. Next we show that $\mu \Phi_{v} u \neq u$ for any $u \in \partial K_{r_{1}}, v \in K$ and $\mu \geq 1$.

If this is not true, then there exist $u_{0} \in \partial K_{r_{1}}$ and $\mu_{0} \geq 1$ such that $\mu_{0} \Phi_{v} u_{0}=$ $u_{0}$. Note that $u_{0}(x)$ satisfies

$$
\left\{\begin{array}{l}
L u_{0}+\mu_{0} h_{1}(x) g_{1}\left(x, u_{0}(x), v(x)\right)=0, \quad x \in I^{\prime}, \\
-\left.\Delta\left(p u_{0}^{\prime}\right)\right|_{x=x_{k}}=\mu_{0} I_{1, k}\left(u_{0}\left(x_{k}\right)\right), \quad k=1,2, \cdots, m \\
\alpha u_{0}(0)-\beta u_{0}^{\prime}(0)=0 \\
\gamma u_{0}(1)+\delta u_{0}^{\prime}(1)=0 .
\end{array}\right.
$$

Multiply equation (3.2) by $\phi_{1}(x)$ and integrate from $a$ to $b$, note that

$$
\begin{aligned}
& \int_{a}^{b} \phi_{1}(x)\left[\left(p(x) u_{0}^{\prime}(x)\right)^{\prime}+q(x) u_{0}(x)\right] d x=\int_{a}^{x_{1}} \phi_{1}(x)\left[\left(p(x) u_{0}^{\prime}(x)\right)^{\prime}\right. \\
& \left.+q(x) u_{0}(x)\right] d x \\
& +\sum_{k=1}^{m-1} \int_{x_{k}}^{x_{k+1}} \phi_{1}(x)\left[\left(p(x) u_{0}^{\prime}(x)\right)^{\prime}+q(x) u_{0}(x)\right] d x
\end{aligned}
$$




$$
\begin{aligned}
& +\int_{x_{m}}^{b} \phi_{1}(x)\left[\left(p(x) u_{0}^{\prime}(x)\right)^{\prime}+q(x) u_{0}(x)\right] d x \\
= & \phi_{1}\left(x_{1}\right) p\left(x_{1}\right) u_{0}^{\prime}\left(x_{1}-0\right)-\int_{a}^{x_{1}} p(x) u_{0}^{\prime}(x) \phi_{1}^{\prime}(x) d x+\int_{a}^{x_{1}} q(x) u_{0}(x) \phi_{1}(x) d x \\
& +\sum_{k=1}^{m-1}\left[\phi_{1}\left(x_{k+1}\right) p\left(x_{k+1}\right) u_{0}^{\prime}\left(x_{k+1}-0\right)-\phi_{1}\left(x_{k}\right) p\left(x_{k}\right) u_{0}^{\prime}\left(x_{k}+0\right)\right. \\
& -\int_{x_{k}}^{x_{k+1}} p(x) u_{0}^{\prime}(x) \phi_{1}^{\prime}(x) d x \\
& \left.+\int_{x_{k}}^{x_{k+1}} q(x) u_{0}(x) \phi_{1}(x) d x\right]-\phi_{1}\left(x_{m}\right) p\left(x_{m}\right) u_{0}^{\prime}\left(x_{m}+0\right) \\
& -\int_{x_{m}}^{b} p(x) u_{0}^{\prime}(x) \phi_{1}^{\prime}(x) d x \\
& +\int_{x_{m}}^{b} q(x) u_{0}(x) \phi_{1}(x) d x \\
= & -\sum_{k=1}^{m} \Delta\left(p\left(x_{k}\right) u_{0}^{\prime}\left(x_{k}\right)\right) \phi_{1}\left(x_{k}\right)-\int_{a}^{b} p(x) \phi_{1}^{\prime}(x) u_{0}^{\prime}(x) d x+\int_{a}^{b} q(x) \phi_{1}(x) u_{0}(x) d x .
\end{aligned}
$$

Also note that

$$
\begin{aligned}
\int_{a}^{b} p(x) \phi_{1}^{\prime}(x) u_{0}^{\prime}(x) d x & =\int_{a}^{b} p(x) \phi_{1}^{\prime}(x) d u_{0}(x)=-\int_{a}^{b} u_{0}(x)\left(p(x) \phi_{1}^{\prime}(x)\right)^{\prime} d x \\
& =\int_{a}^{b} u_{0}(x) q(x) \phi_{1}(x) d x+\lambda_{1} \int_{a}^{b} h_{1}(x) \phi_{1}(x) u_{0}(x) d x
\end{aligned}
$$

$$
\begin{aligned}
\int_{a}^{b} \phi_{1}(x)\left[\left(p(x) u_{0}^{\prime}(x)\right)^{\prime}\right. & \left.+q(x) u_{0}(x)\right] d x \\
= & -\sum_{k=1}^{m} \Delta\left(p\left(x_{k}\right) u_{0}^{\prime}\left(x_{k}\right)\right) \phi_{1}\left(x_{k}\right)-\lambda_{1} \int_{a}^{b} h_{1}(x) \phi_{1}(x) u_{0}(x) d x \\
= & \sum_{k=1}^{m} \mu_{0} I_{1, k}\left(u_{0}\left(x_{k}\right)\right) \phi_{1}\left(x_{k}\right)-\lambda_{1} \int_{a}^{b} u_{0}(x) h_{1}(x) \phi_{1}(x) d x
\end{aligned}
$$

So we obtain

$$
\lambda_{1} \int_{a}^{b} u_{0}(x) h_{1}(x) \phi_{1}(x) d x
$$




$$
\begin{aligned}
= & \sum_{k=1}^{m} \mu_{0} I_{1, k}\left(u_{0}\left(x_{k}\right)\right) \phi_{1}\left(x_{k}\right)+\mu_{0} \int_{a}^{b} \phi_{1}(x) h_{1}(x) g_{1}\left(x, u_{0}(x), v(x)\right) d x \\
\geq & (1-\varepsilon) \sum_{k=1}^{m} u_{0}\left(x_{k}\right) I_{1,0}(k) \phi_{1}\left(x_{k}\right) \\
& +(1-\varepsilon) \inf _{z \in R^{+}} g_{1,0}(z) \int_{a}^{b} \phi_{1}(x) u_{0}(x) h_{1}(x) d x .
\end{aligned}
$$

Since $u_{0}(x) \geq \sigma\left\|u_{0}\right\|=\sigma r_{1}$, we have

$$
\int_{a}^{b} \phi_{1}(x) u_{0}(x) h_{1}(x) d x>0 \text { and } \sum_{k=1}^{m} u_{0}\left(x_{k}\right) I_{1,0}(k) \phi_{1}\left(x_{k}\right)>0 .
$$

So from the above inequality we see that $\lambda_{1}>(1-\varepsilon) \inf _{z \in R^{+}} g_{1,0}(z)$.

Thus

$$
\begin{aligned}
{\left[\lambda_{1}-(1-\varepsilon)\right.} & \left.\inf _{z \in R^{+}} g_{1,0}(z)\right] \int_{a}^{b} u_{0}(x) h_{1}(x) \phi_{1}(x) d x \\
& \geq(1-\varepsilon) \sum_{k=1}^{m} I_{1,0}(k) \phi_{1}\left(x_{k}\right) u_{0}\left(x_{k}\right) \geq(1-\varepsilon) \sigma r_{1} \sum_{k=1}^{m} I_{1,0}(k) \phi_{1}\left(x_{k}\right) .
\end{aligned}
$$

Since $\int_{a}^{b} u_{0}(x) h_{1}(x) \phi_{1}(x) d x \leq r_{1} \int_{a}^{b} \phi_{1}(x) h_{1}(x) d x$, we have

$$
\left[\lambda_{1}-(1-\varepsilon) \inf _{z \in R^{+}} g_{1,0}(z)\right] \int_{a}^{b} h_{1}(x) \phi_{1}(x) d x \geq(1-\varepsilon) \sigma \sum_{k=1}^{m} I_{1,0}(k) \phi_{1}\left(x_{k}\right),
$$

which contradicts (3.2) again. Hence,from Lemma 2.5 we have .

$$
i\left(\Phi, K_{r_{1}}, K\right)=0, \quad \forall v \in K
$$

On the other hand, from $\left(H_{1}\right)$, there exists $H>r_{1}$ such that

$$
\begin{aligned}
g_{1}(x, u, v) \leq\left(g_{1}^{\infty}(v)+\varepsilon\right) u, I_{1, k}(u) \leq\left(I_{1}^{\infty}(k)+\varepsilon\right) u & \\
& \forall x \in[a, b], u \geq H, v \in R^{+} .
\end{aligned}
$$

Let

$$
C=\max _{\substack{a \leq x \leq b \\ 0 \leq u \leq H, v \in R^{+}}}\left(\left|g_{1}(x, u, v)-\left(g_{1}^{\infty}(z)+\varepsilon\right) u\right|+\sum_{k=1}^{m}\left|I_{1, k}(u)-\left(I_{1}^{\infty}(k)+\varepsilon\right) u\right|\right) .
$$


It is clear that

$$
\begin{aligned}
& g_{1}(x, u, v) \leq\left(g_{1}^{\infty}(v)+\varepsilon\right) u+C, I_{1, k}(u) \leq\left(I_{1}^{\infty}(k)+\varepsilon\right) u+C, \\
& \forall x \in[a, b], u \geq 0, v \in R^{+} .
\end{aligned}
$$

Next we show that if $R_{1}$ is large enough, then $\mu \Phi_{v} u \neq u$ for any $u \in \partial K_{R_{1}}$, $v \in K$ and $0<\mu \leq 1$. In fact, if there exist $u_{0} \in \partial K_{R_{1}}$ and $0<\mu_{0} \leq 1$ such that $\mu_{0} \Phi_{v} u_{0}=u_{0}$, then $u_{0}(x)$ satisfies equation (3.2). Multiply equation (3.2) by $\phi_{1}(x)$ and integrate from a to b, using (3.5), to obtain

$$
\begin{aligned}
& \lambda_{1} \int_{a}^{b} u_{0}(x) h_{1}(x) \phi_{1}(x) d x \\
= & \sum_{k=1}^{m} \mu_{0} I_{1, k}\left(u_{0}\left(x_{k}\right)\right) \phi_{1}\left(x_{k}\right)+\mu_{0} \int_{a}^{b} \phi_{1}(x) h_{1}(x) g_{1}\left(x, u_{0}(x), v(x)\right) d x \\
\leq & \sum_{k=1}^{m}\left(I_{1}^{\infty}(k)+\varepsilon\right) \phi_{1}\left(x_{k}\right) u_{0}\left(x_{k}\right)+\int_{0}^{1} \phi_{1}(x) u_{0}(x) h_{1}(x) d x\left(\sup _{z \in R^{+}} g_{1}^{\infty}(z)+\varepsilon\right) \\
+ & C\left(\sum_{k=1}^{m} \phi_{1}\left(x_{k}\right)+\int_{a}^{b} \phi_{1}(x) h_{1}(x) d x\right),
\end{aligned}
$$

i.e.

$$
\begin{aligned}
& \left(\lambda_{1}-\sup _{z \in R^{+}} g_{1}^{\infty}(z)-\varepsilon\right) \int_{a}^{b} u_{0}(x) h_{1}(x) \phi_{1}(x) d x \\
& \quad \leq \sum_{k=1}^{m}\left(I_{1}^{\infty}(k)+\varepsilon\right) \phi_{1}\left(x_{k}\right) u_{0}\left(x_{k}\right)+C\left(\sum_{k=1}^{m} \phi_{1}\left(x_{k}\right)+\int_{a}^{b} \phi_{1}(x) h_{1}(x) d x\right) \\
& \quad \leq\left\|u_{0}\right\| \sum_{k=1}^{m}\left(I_{1}^{\infty}(k)+\varepsilon\right) \phi_{1}\left(x_{k}\right)+C\left(\sum_{k=1}^{m} \phi_{1}\left(x_{k}\right)+\int_{a}^{b} \phi_{1}(x) h_{1}(x) d x\right)
\end{aligned}
$$

Also we have $\int_{a}^{b} u_{0}(x) h_{1}(x) \phi_{1}(x) d x \geq\left\|u_{0}\right\| \int_{a}^{b} \sigma \phi_{1}(x) h_{1}(x) d x$, and this together with (3.6) yields

$$
\left\|u_{0}\right\| \leq \frac{C\left(\sum_{k=1}^{m} \phi_{1}\left(x_{k}\right)+\int_{a}^{b} \phi_{1}(x) h_{1}(x) d x\right)}{\left(\lambda_{1}-\sup _{z \in R^{+}} g_{1}^{\infty}(z)-\varepsilon\right) \int_{a}^{b} \sigma \phi_{1}(x) h_{1}(x) d x-\sum_{k=1}^{m}\left(I_{1}^{\infty}(k)+\varepsilon\right) \phi_{1}\left(x_{k}\right)}=: \bar{R}_{1} .
$$

Let $R_{1}=\max \left\{r_{1}, \bar{R}_{1}\right\}$, then for any $x \in \partial K_{R_{1}}$ and $0<\mu \leq 1$, we have $\mu \Phi_{v} u \neq u$. Thus

$$
i\left(\Phi, K_{R_{1}}, K\right)=1
$$


In view of (3.3) and (3.7), we obtain

$$
i\left(\Phi, K_{R_{1}} \backslash \bar{K}_{r_{1}}, K\right)=1 .
$$

Lemma 3.2. If $\left(H_{2}\right)$ and $\left(H_{3}\right)$ are satisfied, then $i\left(\Psi_{u}, K_{R_{2}} \backslash \bar{K}_{r_{2}}, K\right)=-1$.

Proof. Since $\left(H_{2}\right)$ holds, there exists $0<\varepsilon<\min \left\{\mu_{1}-\sup _{z \in R^{+}} g_{2}^{0}(z), 1\right\}$ such that

$$
\begin{gathered}
\left(\mu_{1}-\varepsilon-\sup _{z \in R^{+}} g_{2}^{0}(z)\right) \int_{a}^{b} \sigma \phi_{2}(x) h_{2}(x) d x>\sum_{k=1}^{m}\left(I_{2}^{0}(k)+\varepsilon\right) \phi_{2}\left(x_{k}\right), \\
(1-\varepsilon)\left[\inf _{z \in R^{+}} g_{2, \infty}(z)+\frac{\sigma \sum_{k=1}^{m} I_{2, \infty}(k) \phi_{2}\left(x_{k}\right)}{\int_{a}^{b} \phi_{2}(x) h_{2}(x) d x}\right]>\mu_{1} .
\end{gathered}
$$

One can find $r_{0}>0$ such that

$g_{2}(x, v, u) \leq\left(g_{2}^{0}(u)+\varepsilon\right) v, I_{2, k}(v) \leq\left(I_{2}^{0}(k)+\varepsilon\right) v, \forall x \in[a, b], 0 \leq v \leq r_{0}, u \in R^{+}$.

Let $r_{2} \in\left(0, r_{0}\right)$. Now we prove that $\mu \Psi_{u} v \neq v$ for any $v \in \partial K_{r_{2}}, u \in K$ and $0<\mu \leq 1$. If this is not true, then there exist $v_{0} \in \partial K_{r_{2}}$ and $0<\mu_{0} \leq 1$ such that $\mu_{0} \Psi_{u} v_{0}=v_{0}$. Note that $v_{0}(x)$ satisfies

$$
\left\{\begin{array}{l}
L v_{0}+\mu_{0} h_{2}(x) g_{2}\left(x, v_{0}(x), u(x)\right)=0, \quad x \in I^{\prime}, \\
-\left.\Delta\left(p v_{0}^{\prime}\right)\right|_{x=x_{k}}=\mu_{0} I_{2, k}\left(v_{0}\left(x_{k}\right)\right), \quad k=1,2, \cdots, m \\
\alpha v_{0}(0)-\beta v_{0}^{\prime}(0)=0 \\
\gamma v_{0}(1)+\delta v_{0}^{\prime}(1)=0
\end{array}\right.
$$

Multiply equation (3.9) by $\phi_{2}(x)$ and integrate from a to b,and proceeding as in the proof of Lemma 3.1, we have

$$
\begin{aligned}
& \mu_{1} \int_{a}^{b} v_{0}(x) h_{2}(x) \phi_{2}(x) d x \\
= & \sum_{k=1}^{m} \mu_{0} I_{2, k}\left(v_{0}\left(x_{k}\right)\right) \phi_{2}\left(x_{k}\right)+\mu_{0} \int_{a}^{b} \phi_{2}(x) h_{2}(x) g_{2}\left(x, v_{0}(x), u(x)\right) d x \\
\leq & \sum_{k=1}^{m}\left(I_{2}^{0}(k)+\varepsilon\right) v_{0}\left(x_{k}\right) \phi_{2}\left(x_{k}\right)+\int_{a}^{b} \phi_{2}(x) v_{0}(x) h_{2}(x) d x\left(\sup _{z \in R^{+}} g_{2}^{0}(z)+\varepsilon\right)
\end{aligned}
$$


Since $v_{0}(x) \geq \sigma\left\|v_{0}\right\|=\sigma r_{2}$, for $x \in[a, b]$, we have

$$
\begin{aligned}
& r_{2}\left(\mu_{1}-\sup _{z \in R^{+}} g_{2}^{0}(z)-\varepsilon\right) \int_{a}^{b} \sigma h_{2}(x) \phi_{2}(x) d x \\
\leq & \left(\mu_{1}-\sup _{z \in R^{+}} g_{2}^{0}(z)-\varepsilon\right) \int_{a}^{b} v_{0}(x) h_{2}(x) \phi_{2}(x) d x \\
\leq & \sum_{k=1}^{m}\left(I_{2}^{0}(k)+\varepsilon\right) \phi_{2}\left(x_{k}\right) v_{0}\left(x_{k}\right) \\
\leq & r_{2} \sum_{k=1}^{m}\left(I_{2}^{0}(k)+\varepsilon\right) \phi_{2}\left(x_{k}\right) .
\end{aligned}
$$

which is a contradiction with (3.8). By Lemma 2.4, we have

$$
i\left(\Psi_{u}, K_{r_{2}}, K\right)=1 .
$$

On the other hand, from $\left(H_{2}\right)$, there exists $H>r_{2}$ such that

$$
\begin{gathered}
g_{2}(x, v, u) \geq g_{2, \infty}(u)(1-\varepsilon) v, I_{2, k}(v) \geq I_{2, \infty}(k)(1-\varepsilon) v, \\
\forall x \in[a, b], v \geq H, u \in R^{+} .
\end{gathered}
$$

Let

$$
\begin{aligned}
C= & \max _{\substack{a \leq x \leq b, 0 \leq v \leq H, u \in R^{+}}}\left(\left|g_{2}(x, v, u)-g_{2, \infty}(u)(1-\varepsilon) v\right|\right. \\
& \left.+\sum_{k=1}^{m}\left|I_{2, k}(v)-I_{2, \infty}(k)(1-\varepsilon) v\right|\right) .
\end{aligned}
$$

It is clear that

$$
\begin{gathered}
g_{2}(x, v, u) \geq g_{2, \infty}(u)(1-\varepsilon) v-C, \quad I_{2, k}(v) \geq I_{2, \infty}(k)(1-\varepsilon) v-C, \\
\forall x \in[a, b], v \geq 0, u \in R^{+} .
\end{gathered}
$$

Choose $R_{2}>R_{0}:=\max \left\{\frac{H}{\sigma}, r_{2}\right\}$ and let $v \in \partial K_{R_{2}}, u \in K$. Since $v(x) \geq$ $\sigma\|v\|=\sigma R_{2}>H$ for $x \in[a, b], \quad u \in K$, from (3.11) we see that

$$
\begin{gathered}
g_{2}(x, v(x), u(x)) \geq g_{2, \infty}(u(x))(1-\varepsilon) v(x) \geq \sigma g_{2, \infty}(u(x))(1-\varepsilon) R_{2}, \\
I_{2, k}\left(v\left(x_{k}\right) \geq \sigma I_{2, \infty}(k)(1-\varepsilon) R_{2} .\right.
\end{gathered}
$$


Essentially the same reasoning as above yields $\inf _{v \in \partial K_{R_{2}}}\left\|\Psi_{u} v\right\|>0$. Next we show that if $R_{2}$ is large enough, then $\mu \Psi_{u} v \neq v$ for any $v_{0} \in \partial K_{R_{2}}$ and $\mu \geq 1$. In fact, if there exist $v_{0} \in \partial K_{R_{2}}$ and $\mu_{0} \geq 1$ such that $\mu_{0} \Psi_{u} v_{0}=v_{0}$, then $v_{0}(x)$ satisfies equation (3.9) . Multiply equation (3.9) by $\phi_{2}(x)$ and integrate from a to $b$, using integration by parts in the left side to obtain

$$
\begin{aligned}
& \mu_{1} \int_{a}^{b} v_{0}(x) h_{2}(x) \phi_{2}(x) d x \\
= & \sum_{k=1}^{m} \mu_{0} I_{2, k}\left(v_{0}\left(x_{k}\right)\right) \phi_{2}\left(x_{k}\right)+\mu_{0} \int_{a}^{b} \phi_{2}(x) h_{2}(x) g_{2}\left(x, v_{0}(x), u(x)\right) d x \\
\geq & (1-\varepsilon) \sum_{k=1}^{m} I_{2, \infty}(k) \phi_{2}\left(x_{k}\right) v_{0}\left(x_{k}\right) \\
& +(1-\varepsilon) \inf _{z \in R^{+}} g_{2, \infty}(z) \int_{a}^{b} v_{0}(x) \phi_{2}(x) h_{2}(x) d x \\
& -C\left(\sum_{k=1}^{m} \phi_{2}\left(x_{k}\right)+\int_{a}^{b} \phi_{2}(x) h_{2}(x) d x\right) .
\end{aligned}
$$

If $\inf _{z \in R^{+}} g_{2, \infty}(z) \leq \mu_{1}$, then we have

$$
\begin{aligned}
& {\left[\mu_{1}-(1-\varepsilon) \inf _{z \in R^{+}} g_{2, \infty}(z)\right] \int_{a}^{b} v_{0}(x) h_{2}(x) \phi_{2}(x) d x} \\
& \quad+C\left(\sum_{k=1}^{m} \phi_{2}\left(x_{k}\right)+\int_{a}^{b} \phi_{2}(x) h_{2}(x) d x\right) \geq(1-\varepsilon) \sum_{k=1}^{m} I_{2, \infty}(k) \phi_{2}\left(x_{k}\right) v_{0}\left(x_{k}\right) .
\end{aligned}
$$

thus

$$
\begin{aligned}
& \left\|v_{0}\right\|\left[\mu_{1}-(1-\varepsilon) \inf _{z \in R^{+}} g_{2, \infty}(z)\right] \int_{a}^{b} h_{2}(x) \phi_{2}(x) d x \\
& \quad+C\left(\sum_{k=1}^{m} \phi_{2}\left(x_{k}\right)+\int_{a}^{b} \phi_{2}(x) h_{2}(x) d x\right) \geq(1-\varepsilon) \sigma\left\|v_{0}\right\| \sum_{k=1}^{m} I_{2, \infty}(k) \phi_{2}\left(x_{k}\right) .
\end{aligned}
$$

and

$$
\begin{aligned}
& \left\|v_{0}\right\| \leq \\
& \frac{C\left(\sum_{k=1}^{m} \phi_{2}\left(x_{k}\right)+\int_{a}^{b} \phi_{2}(x) h_{2}(x) d x\right)}{(1-\varepsilon) \sigma \sum_{k=1}^{m} I_{2, \infty}(k) \phi_{2}\left(x_{k}\right)-\left[\mu_{1}-(1-\varepsilon) \inf _{z \in R^{+}} g_{2, \infty}(z)\right] \int_{a}^{b} \phi_{2}(x) h_{2}(x) d x}
\end{aligned}
$$




$$
=: \bar{R}_{2} \text {. }
$$

If $\inf _{z \in R^{+}} g_{2, \infty}(z)>\mu_{1}$, we can choose $\varepsilon>0$ such that $(1-\varepsilon) \inf _{z \in R^{+}} g_{2, \infty}(z)>\mu_{1}$, then we have

$$
\begin{aligned}
& C\left(\sum_{k=1}^{m} \phi_{2}\left(x_{k}\right)+\int_{a}^{b} \phi_{2}(x) h_{2}(x) d x\right) \\
& \geq\left[(1-\varepsilon) \inf _{z \in R^{+}} g_{2, \infty}(z)-\mu_{1}\right] \int_{a}^{b} \phi_{2}(x) v_{0}(x) h_{2}(x) d x \\
& \quad \geq\left[(1-\varepsilon) \inf _{z \in R^{+}} g_{2, \infty}(z)-\mu_{1}\right] \sigma\left\|v_{0}\right\| \int_{a}^{b} \phi_{2}(x) h_{2}(x) d x .
\end{aligned}
$$

Thus

$$
\left\|v_{0}\right\| \leq \frac{C\left(\sum_{k=1}^{m} \phi_{2}\left(x_{k}\right)+\int_{a}^{b} \phi_{2}(x) h_{2}(x) d x\right)}{\left[(1-\varepsilon) \inf _{z \in R^{+}} g_{2, \infty}(z)-\mu_{1}\right] \sigma \int_{a}^{b} \phi_{2}(x) h_{2}(x) d x}=: \bar{R}_{2} .
$$

Let $R_{2}>\max \left\{r_{2}, \bar{R}_{2}\right\}$, then for any $v \in \partial K_{R_{2}}, u \in K$ and $\mu \geq 1$, we have $\mu \Psi_{u} v \neq v$. Hence hypothesis (ii) of Lemma 2.5 is satisfied and

$$
i\left(\Psi_{u}, K_{R_{2}}, K\right)=0 .
$$

In view of (3.10) and (3.14), we obtain

$$
i\left(\Psi_{u}, K_{R_{2}} \backslash \bar{K}_{r_{2}}, K\right)=-1
$$

Proof of Theorem 3.1. Since $\left(H_{1}\right)-\left(H_{3}\right)$ are satisfied ,from Lemma2.3 we get $\Phi_{v}: K \rightarrow K, \Psi_{u}: K \rightarrow K$ and $T: K \times K \rightarrow K \times K$ are completely continuous. From Lemma3.1,3.2 and 2.6 we have

$$
\begin{array}{r}
i\left(T, K_{R_{1}} \backslash \bar{K}_{r_{1}} \times K_{R_{2}} \backslash \bar{K}_{r_{2}}, K \times K\right)=i\left(\Phi_{v}, K_{R_{1}} \backslash \bar{K}_{r_{1}}, K\right) \times i\left(\Psi_{u}, K_{R_{2}} \backslash \bar{K}_{r_{2}}, K\right) \\
=-1 .
\end{array}
$$

Thus, system (1.1)has at least one solution $(\mathrm{u}, \mathrm{v})$.

Corollary 3.1. The conclusion of Theorem 3.1 is valid if $\left(H_{1}\right)$ and $\left(H_{2}\right)$ are replaced by

$$
\left(H_{1}^{*}\right) \inf _{z \in R^{+}} g_{1,0}(z)=\infty \text { or } \sum_{k=1}^{m} I_{1,0}(k) \phi_{1}\left(x_{k}\right)=\infty,
$$




$$
\begin{gathered}
\sup _{z \in R^{+}} g_{1}^{\infty}(z)=0 \text { and } I_{1}^{\infty}(k)=0, k=1,2, \ldots m . \\
\left(H_{2}^{*}\right) \sup _{z \in R^{+}} g_{2}^{0}(z)=0 \text { and } I_{2}^{0}(k)=0, k=1,2, \ldots m ; \\
\inf _{z \in R^{+}} g_{2, \infty}(z)=\infty \text { or } \sum_{k=1}^{m} I_{2, \infty}(k) \phi_{2}\left(x_{k}\right)=\infty .
\end{gathered}
$$

\section{Acknowledgments}

Project supported by Heilongjiang province education department natural science research item, China (12541076).

\section{References}

[1] X. Y. Cheng, C. K. Zhong, Existence of positive solutions for a secondorder ordinary differential system, J. Math. Anal. Appl. 312 (2005), 14-23.

[2] W. Ding, M. Han, Periodic boundary value problem for the second order impulsive functional differential equations, Appl. Math. Comput. 155 (2004), 709-726.

[3] D. Guo and V. Lakshmikantham, Nonlinear Problems in Abstract Cones, Academic Press, Boston, MA, 1988.

[4] D. Guo, J. Sun, Z. Liu, Functional Methods in Nonlinear Ordinary Differential Equations, Shandong Science and Technology Press, Jinan, 1995 (in Chinese).

[5] S. G. Hristova, D. D. Bainov, Monotone-iterative techniques of V. Lakshmikantham for a boundary value problem for systems of impulsive differential-difference equations, J. Math. Anal. Appl. 197 (1996), 1-13.

[6] V. Lakshmikntham, D. D. Bainov and P. S. Simeonov, Theory of Impulsive Differential Equations, World Scientific, Singapore, (1989).

[7] E. Lee, Y. Lee, Multiple positive solutions of singular two point boundary value problems for second order impulsive differential equation, Appl. Math. Comput. 158 (2004), 745-759. 
[8] X. Lin, D. Jiang, Multiple positive solutions of Dirchlet boundary value problems for second order impulsive differential equations, J. Math. Anal. Appl. In press.

[9] X. Liu, D. Guo, Periodic Boundary value problems for a class of secondOrder impulsive integro-differential equations in Banach spaces, J. Math. Anal. Appl. 216 (1997), 284-302.

[10] I. Rachunkova, J. Tomecek, Impulsive BVPs with nonlinear boundary conditions for the second order differential equations without growth restrictions, J. Math. Anal. Appl. 292 (2004), 525-539. 
